\title{
Genetic Algorithms for Polycentric Curves Interpretation
}

\author{
Emanuela Lanzara \\ Mara Capone
}

\section{Abstract}

This research work into Evolutionary Computing field aims at improving a dataset of algorithmic generative definitions able to return an optimized 'semi-ideal' curve that best fits a generic reality-based profile, starting from some of its points. This paper shows GAs_Genetic Algorithms applications especially with regards to study, interpretation and definition of generic polycentric curves. Current VPL tools (Galapagos-Rhino, McNeel) allow to test Evolutionary Theories for problem solving and decision making in architectural research field. According to a human driven approach, an operator defines GENOME, FUNCTION and FITNESS to drive the Evolutionary Solver towards optimized solutions. Some case studies from Historical/Existent Architectural Heritage are used to show how GAs can simplify the digitalization process and big data interpretation.

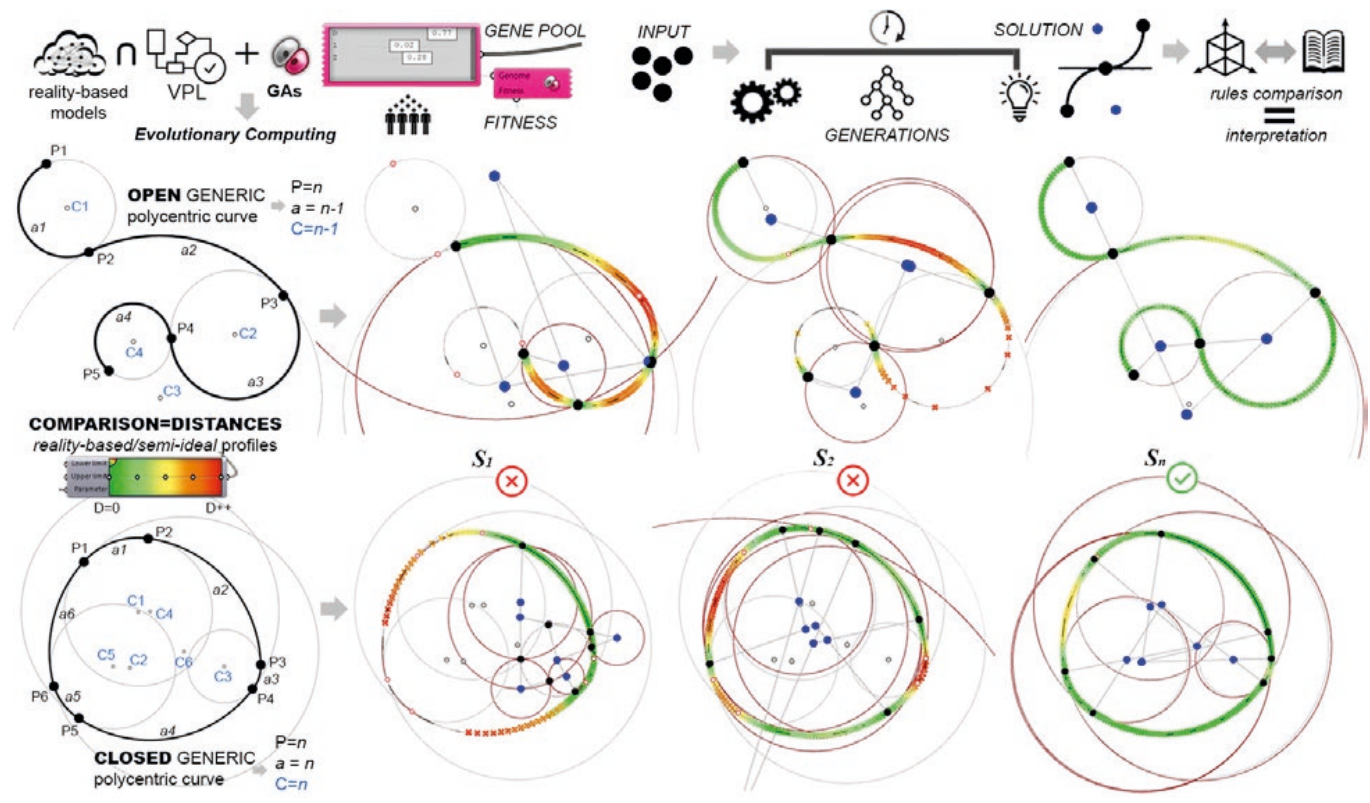




\section{Introduction}

This research work into EC_Evolutionary Computing field aims at developing a dataset of algorithmic generative definitions (VPL), to return optimized 'semi-ideal' curves that best fit reality-based profiles (generic polycentric curves from point cloud segments). This paper especially shows testing of GAs [Holland 1992] for vaulted systems study. EC is a subfield of Al aimed at iterative, continuous and combinatorial search for optimized solutions.

In architectural and engineering fields, it is possible to distinguish applications about optimization of architectural-urban design [Buffi et al. 2020; Canestrino et al. 2020, Palma et al. 2020], analytical-structural applications [Grillanda et al. 20 I7, Khan 20 I5], manufacturing complex elements-systems [Zaremba 20 16; Coutinho 20 I0; Limonge et al. 20 I0], analytical-geometric applications to rebuild and compare shapes [Bianconi et al. 20 I8] and, more specifically, about ovals interpretation [Santagati et al. 20।8].

Moreover, similar approaches are managed with different tools to optimize the curves and surfaces interpretation that describe historical architectural elements, according to stylistic features of cultural heritages and geographical contexts [Samper et al. 2020; Lanzara et al. 2019].

These approaches are also potentially aimed at supporting Al processes [Sim 2020]. This VPL algorithmic definition allows to construct all kind of profile starting from realitybased elements: the only input parameter is the points number. It is the main advantage of the process.

\section{Methodology and Tools}

Digitizalization of existent architectural elements is a process to provide a system of 3D models and related information (parameters/geometric variables, construction techniques, materials). About Historic Heritage, these properties can be extrapolated through a direct analysis of architectural elements (survey) or from specific treatises and historical manuals rules. The parameterization of pointed arches and polycentric curves (vertical sections), used to generate the revolution pointed domes, is one of tested approaches [Capone et al. $2019 a]$. Digital translation of geometric-mathematical rules simplifies the parameterization (geometric genesis) of complex architectural systems and allows to model variable and adaptable configurations [Capone et al. 2019b]. Another approach is based on VPL models built on reality-based profiles used as "input parameters", to compose the wireframe of the architectural element, providing a 'semi-ideal' model closer to the real element. Then, different approaches and tools (VPL/C++) were compared to identify ideal curves and surfaces that best fit point clouds segments [Lanzara et al. 20 I9].

We have applied these approaches to define generic polycentric curves, open or closed, from reality-based profiles using GAs. This contribute shows applications about closed symmetrical polycentric curves and open polycentric curves that could be domes profiles. Ovoidal domes can be generate as revolution surfaces or they can be shaped from curves network. The axes dimensions are not sufficient as input parameters to model these shapes. In fact, it is not uniquely possible to identify and to draw the specific oval that best describes a reality-based profile according to the lengths of its axes only. Starting from the same pair of axes, it is possible to generate infinite ovals and one ellipse [Dotto 2002, p. I4].

Current VPL generative-algorithmic tools (Galapagos-Gh component, Octopus-Gh plugin, Rhino, McNeel) allow to test Evolutionary Theory (Darwin 1859) to support problem solving and decision making processes. According to a human driven approach, the parameters identification to define GENOME, FUNCTION and FITNESS allows to drive the Evolutionary Solver towards an optimized solution.

GAs calculates the optimal position of the end-points (GENE POOL) of a PolyArc (VPL component aimed at built a sequence of tangential continuous circular segments) along a reality-based profile to extrapolate a 'semi-ideal' polycentric curve. A direct comparison between the 'semi-ideal' curves (GA) and the ideal curves (rules) allows to establish which type of ideal profile best fits and decodes the reference curve. The number of points can 
be random or deriving from a critical interpretation of the reference subject. Symmetrical distributions (entrances, chapels, mosaics, niches or structural-decorative elements) simplifies the decomposition of a PolyArc into its segments and allows to define hypotheses about the specific oval profile. If the curve is closed, the points number (GENE POOL) is the same of the arcs number; if it is open, the number of points is the same of the number of arches/centers $+I$.

The Evolutionary Solver combines points by minimizing the distance (Mass Addition)between reality-based curve and PolyArc (FITNESS) to optimize their overlap. FITNESS defined and tested to select the optimal solutions, allow to minimize the sum of the distances between the ideal curve and the reference one and the average distance between curves and they allow to maximize the identification of points whose distance from the reference curve is smaller or equal to a given limit value. A chromatic gradient distinguishes the points along reality-based profile according to their distance from defined 'semi-ideal' curve and/ or the ideal configuration that best fits it: for a value of 0 , points are green; for higher values, points are red.

Once the ideal circular segments of the PolyArc have been identified, it is automatically possible to extract the whole circumferences and the position of their centers for each circular segment. Finally, it is also possible to compare the defined 'semi-ideal' PolyArc and its distributive layout of the centers with ideal profiles (curves built starting from centers along diameters).

The main advantage of this definition is to use the points number as the only input parameter: the difference between closed and open 'semi-ideal' polycentric curves is to communicate this condition by simply using a Boolean Toggle (True/False). Therefore, a single definition allows to analyze, interpret and define a generic open or closed polycentric profile. Another important advantage is to use the same GENE POOL (points along reality-based curve) to generate different curves. The main advance, in itinere, of this research activity is also about study and definition of analytic curves, e.g. conics, only starting from points along reality-based profile.

Fig. I. GA to interpret and defne CLOSEL POLYARC - OVAL profiles (impost and intermediate sections of the Church of S. Giovanni Maggiore's dome. On the right-top: comparison between 'semi-ideal' profile and rules; on the rightbottom: comparison between reality-based profiles, oval and ellipse, (authors elaborations)

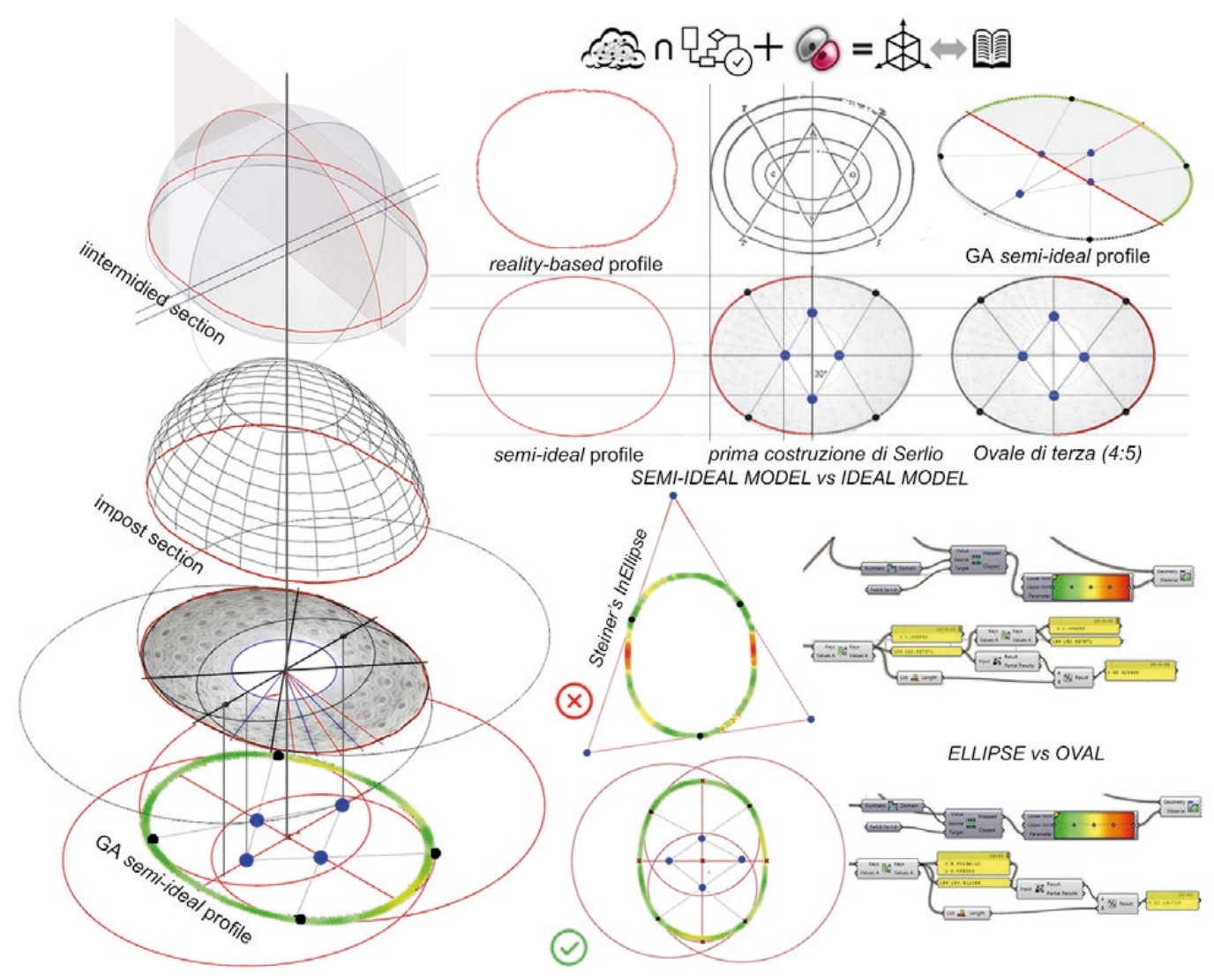




\section{Applications}

GA for OVAL interpretation has been tested to verify the impost and the intermediate sections of the ovoidal coffered dome of the Basilica of S. Giovanni Maggiore in Naples. A double symmetrical oval (four centers) is clearly recognizable with the naked eye by observing the extrusion profile of the molded frame crowning the drum and corresponding to the impost curve of the coffered intrados of the dome. However, the intermediate oval profiles are similar to ellipses.

Although ellipse and oval are conceptually and analytically different curves, for centuries they generate a "conflict" (Migliari 1995): the main reason lies in their formal similarity, often causing interpretative misunderstandings about the attribution of these shapes to geometric elements.

Figure I shows comparisons between 'semi-ideal' ovals, Serlio's rules (I584) and the elliptic profile that best fits the intermediate sections. Unlike the impost profile, the algorithm calculates a minimal difference between intermediate sections and ellipse. However, also the presence of lacunars would confirm the oval shape for the intermediate sections: in fact, oval allows regular offset.

About modeling of hemispherical pointed domes characterized by circular section, we have translated in VPL the geometric rules provided by Serlio and by Palladio; for polycentric vertical sections (curves composed by a series of continuous arches in tangency and curvature), the rules provided by Fontana and by Scamozzi; for pointed arches (different ratios between 'arrow' and radius), the rule illustrated by Vittone [Capone et al. 20 19a]. Therefore, this comparative approach was tested on a series of revolution domes of Historic Architectural Heritage in Naples.

In particular, GA to interpret and build POLICENTRIC ARCHES was tested on the pointed dome of the Church of S. Caterina a Formiello to define the layouts of centers subtending one of its vertical section (without 'vertex').

By comparing Serlio, Fontana, Vittone and Scamozzi's rules and the reality-based sections, we can state that the reality-based profile does not match any of the constructions of historical treatises actually translated into VPL, neither the ellipse. However, the difference between reality-based profiles and ideal curves is not minimal: therefore, it does not depend only on structural problems or constructive irregularities, but also on different stylistic approaches (fig. 2).

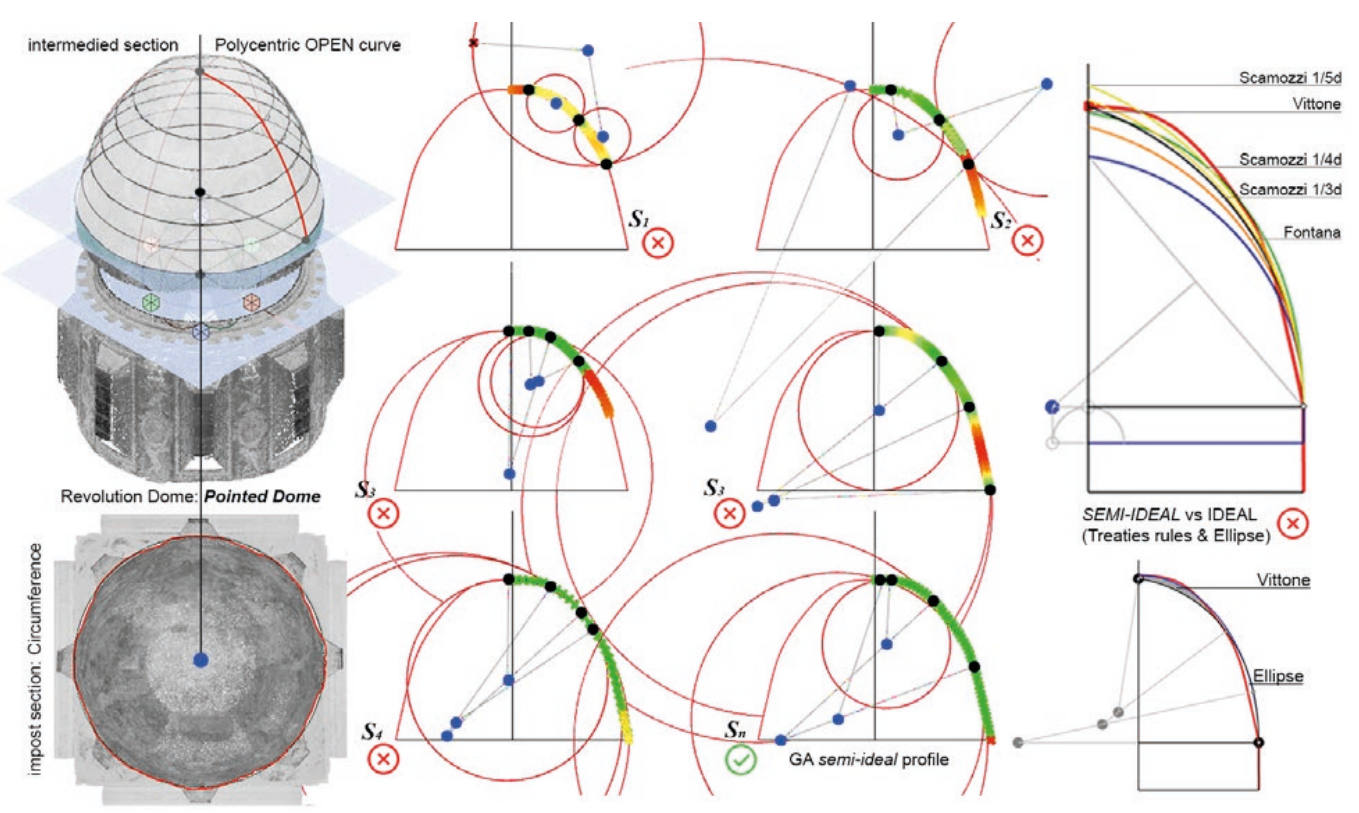




\section{Conclusions and Future Works}

The main progress (in itinere) of this study is to test GAs to interpret and define analytical curves (e.g. conics) and generic profiles characterizing also modern and contemporary architecture. In addition, other future advances can be about deepening the accuracy of the models, improving current VPL definitions and testing other types of algorithms [Gatti 2020]. The semi-automatic interpretation of complex elements simplifies their parameterization according to interoperable logics (VPLBIM-HBIM). Furthermore, this approach is also aimed to test geometric-speculative approaches and to inspire studies with different goals.

\section{References}

Bianconi Fabio, Filippucci Marco, Meconi Magi Federica (2018). Parametrical Vitruvius. Generative modeling of the architectural orders. In SCIRES - SClentific RESearch and Information Technology, 8 (2), pp. $29-48$.

Buffi Alessandro, Bianconi Fabio, Filippucci Marco (2020). Optimization and Evolution in Architectural Morphogenesis: Evolutionary Principles Applied to Mass Housing. In Mehdi Khosrow-Pour (ed.). Research Anthology on Multi-Industry Uses of Genetic Programming and Algorithms. Hershey: IGI Global, pp. 997-I0I6.

Canestrino Giuseppe, Laura Greco, Spada Francesco, Lucente Roberta (2020). Generating architectural plan with evolutionary multiobjective optimization algorithms: a benchmark case with an existent construction system. In Escobar Natalia Builes, Torreblanca-Díaz David A. (eds.). SIGraDi 2020.Transformative Design. XXIV International Conference of the Iberoamerican Society of Digital Graphics, pp. I49- 156

Capone Mara, Lanzara Emanuela (2019.a). Scan-to-Bim vs 3D Ideal Model. HBIM: Parametric tools to study Domes Geometry. In International Archives of Photogrammetry, Remote Sensing and Spatial Information Sciences, vol. XLII-2M9, pp. 21 9-226.

Capone Mara, Lanzara Emanuela (2019.b). 3D data interpretation using treatises geometric rules to built coffered domes. In International Archives of Photogrammetry, Remote Sensing and Spatial Information Sciences, Vol. XLII-2/WI 5, pp. 23 I-238.

Darwin Charles ( 1859). On the Origin of Species by Means of Natural Selection. London: John Murray.

Dotto Edoardo (2002). II Disegno degli ovali armonici. Catania: Le Nove Muse Editrice.

Gatti Nicola, (12 marzo 2020). Matematica e Tech. Algoritmi, facciamo chiarezza sui diversi tipi. Network Digital 360. agendadigitale.eu/cultura-digitale/algoritmi-quale-tipo-per-quale-problema-facciamo-chiarezza/ [04 March 202 I].

Grillanda Nicola, Manconi Fabio, Stochino Fabio, Cazzani Antonio, Bondi Francesco, Chiozzi Andrea, Tralli Antonio (2017) On the analysis of the stellar vault of Santa Maria del Monte in Cagliari. In Proceedings of the International Conference of Computational Methods in Sciences and Engineering (ICCMSE-20 I7). AIP Publishing.

Holland James (1992). Adaptation in Natural and Aritificial Systems: An Introductory Analysis with Applications to Biology, Control and Artificial Intelligence. Cambridge, MA, United States: MIT Press.

Khan Azhar (2015). compdesignstudio.com. Polyline Curve to Arcs-Optimization using Galapagos. youtube.com/ watch?v=03VTBaZk5j0 [23 February 202I]

Lanzara Emanuela, Samper Albert e Herrera Blas (2019). Point Cloud Segmentation and Filtering to verify the geometric genesis of simple and composed vaults. In International Archives of the Photogrammetry, Remote Sensing and Spatial Information Sciences, vol. XLII-2MI5, pp. 645-652.

Lemonge Alfonso C. C., Barbosa, Helio J. C., da Fonseca Leonardo G. and Coutinho, Alvaro L. G. A. (20 I0). A genetic algorithm for topology optimization of dome structures. In Proceedings of 2nd International Conference on Engineering Optimization, pp. I- I5.

Migliari Riccardo, 1995. Ellissi e ovali, epilogo di un conflitto. In Palladio 16, 1995. riccardo.migliari.it/pdf_saggi//995_ellissi_ovali_ Ir.pdf [23 February 202I].

Palma Valerio, Accorsi Federico, Casasso Alessandro, Bianco Carlo, Cutrì Sarah, Robiglio Matteo, Tosco Tiziana (2020). AdRem: An Integrated Approach for Adaptive Remediation. In Sustainability 202 I, I3, 28. p. I-I5.

Samper Albert, Herrera Blas, González Genaro. (2020). Geometrical review of the dome in Palau Güell. Revisión de la tipología geométrica de la cúpula del Palau Güell. In Informes de la Construcción, 72 (558).

Santagati Cettina, La Russa Federico Mario, Galizia Mariateresa, Magnano di San Lio Eugenio (2018). Towards a generic parametric algorithm for the geometric investigation of Baroque Oval plans: an application on Sicilian cases. In Williams Kim, Bevilacqua Marco Giorgio (eds). Nexus 20 I 8. Architecture and Mathematics. Torino: KWB, pp. 245-250.

Sim Victor (2020). Using Genetic Algorithms to Train Neural Networks. In Towards Data Science. towardsdatascience.com/ using-genetic-algorithms-to-train-neural-networks-b5ffe0d5 I32 I ( 2 February 202 I).

\section{Authors}

Emanuela Lanzara, Dept. of Structure for Engineering and Architecture, University Federico II of Naples, emanuela.lanzara@unina.it Mara Capone, Dept. of Architecture, University Federico II of Naples, mara.capone@unina.it 
